International Journal of Distributed and Parallel Systems (IJDPS) Vol.3, No.3, May 2012

\title{
STACKED PRINTEd ANTENNAS ARRAY FOR C BAND APPLICATIONS
}

\author{
M. S. Bahloul, M. Abri and F. T. Bendimerad \\ Laboratoire de Télécommunications, Département de Génie Electrique \\ Faculté de Technologie, Université Abou-Bekr Belkaïd -Tlemcen \\ BP 230, Pôle Chetouane, 13000 Tlemcen- Algérie \\ salimttleyahoo.fr
}

\begin{abstract}
The work presented in this paper proposes a technique for bandwidth enhancement by using log periodic printed antennas arrays. To achieve this, an analysis rigorous method (Moment's method) will be retained to validate the antennas array performances. For this, antennas arrays operating in $C$ band with linear polarization has been studied. These last, present a good performances in adaptation and polarization. The radiating elements used are multi-layered type supplied through a slot in the ground plane. The presented simulation results confirm the validity of the proposed technique.
\end{abstract}

\section{KEY WORDS}

Antennas array, Log periodic concept, analysis, synthesis, optimization, design, Moment's method, ADS/Momentum.

\section{INTRODUCTION}

Modern technologies are directed towards the miniaturization of antennas while trying to keep the best performances, the printed antenna is designed to satisfy these needs, it is a conductive metal of particular form placed on a substrate finished by a ground plane, its miniature character makes it possible to integrate it easily in emission and reception systems. A printed antenna presents a weak band-width and gain, association in array of several printed antennas makes it possible to compensate the single antenna limitations characteristic and to improve their gain and radiation

performances, although their design is difficult because of their electromagnetic structure complexity, these antenna have the advantage of being able to be manufactured in great quantity with very weak cost. One of the antennas disadvantages remains a narrow band-width.

The interest in broadband systems is confirmed every day. The multiplicity of standards for future terminals, the exploration of microwave signals in the medical field, the detector development of anti-personnel mines, require the use of broadband antenna [1] [2]. Many antennas use a wide frequency range, from these log periodic antennas which are defined by angles or by multiple elements that increase steadily in geometrical progression. These antennas are characterized by their frequency independency, by a wide bandwidth, constant impedance over the entire frequency range, maximum gain and good directivity.

The objective of this work is to design log periodic printed antennas array functioning in the $\mathrm{C}$ Band using the method of moments of momentum Software in order to increase the bandwidth. 


\section{Formulation AND Design}

\section{1. Log-periodic antenna basic principle}

The log periodic antennas as shown in figure 1 is a radiating structures transformed into a similar structure by a multiplication of its dimensions by a factor $\tau$, it has the same properties, one at the frequency $f$, and the other at the frequency $f / \tau$.

In general, the geometry of any antenna is the multiplicity of the adjacent one, there is a relationship between them remains constant throughout structure. If $\mathrm{L}_{\mathrm{n}}$ represents a dimension of the $n$th cell and $L_{n+1}$ is that of $(n+1)$ th cells, then the relationship is:

$$
\frac{\mathrm{L}_{\mathrm{n}+1}}{\mathrm{~L}_{\mathrm{n}}}=\tau
$$

The log-periodic structure is the result of a cell size from one period to another, from where the performance is periodic with the logarithm of the frequency [3-6].

The length $\mathrm{L}$ and width $\mathrm{W}$ of the patch are connected by the factor $\tau$ :

$$
\tau=\frac{\mathrm{L}_{\mathrm{n}+1}}{\mathrm{~L}_{\mathrm{n}}}=\frac{\mathrm{W}_{\mathrm{n}+1}}{\mathrm{~W}_{\mathrm{n}}}
$$

If we multiply all the dimensions of the array by the factor $\tau$, the element becomes $(m+1)$ and the element $(m+1)$ becomes an element $(m+2)$.

Consequently, the arrays have the same properties of radiation at all frequencies which are connected by the factor $\tau$.

$$
\mathrm{f}_{1}, \mathrm{f}_{2}=\tau . \mathrm{f}_{1}, \mathrm{f}_{3}=\tau^{2} \mathrm{f}_{1}, \mathrm{f}_{4}=\tau^{3} \mathrm{f}_{1}
$$

It is noted that:

$$
\ln \frac{\mathrm{f}_{2}}{\mathrm{f}_{1}}=\ln \tau ; \ln \frac{\mathrm{f}_{3}}{\mathrm{f}_{1}}=2 \ln \tau
$$

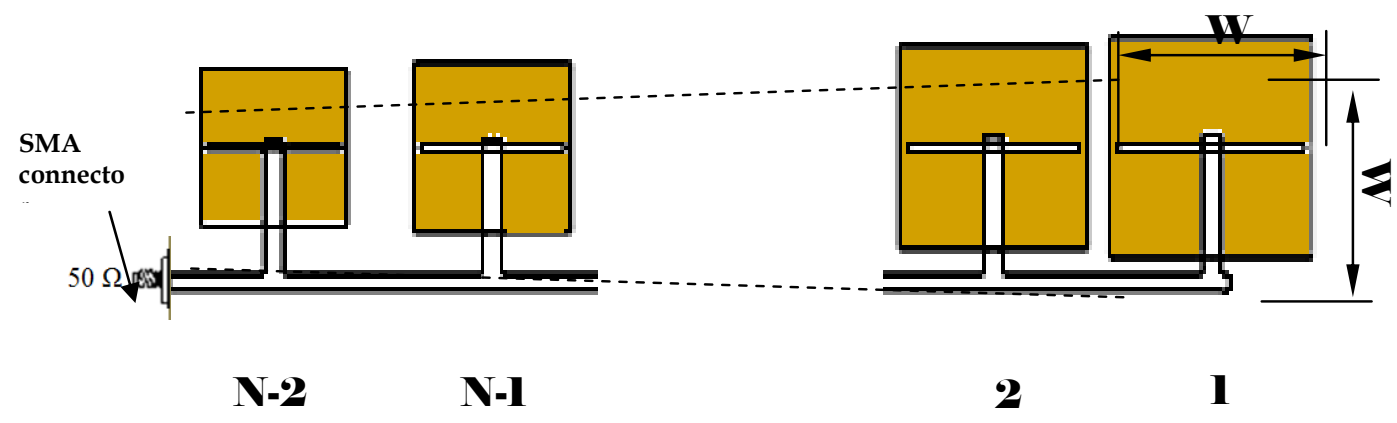

Figure 1. Log periodic antenna array architecture. 


\section{2. Multilayerd antenna}

In general, a printed antenna can be fed either by a coaxial probe, or by microstrip line in the plane of the radiating element.

In this case, a stray radiation of the supply line is added to that of the antenna. To resolve this problem, a new feed configuration is proposed as shown in Figure 2. It involves a separation between the antenna and the microstrip line by a ground plane. An air layer is inserted between the ground plane and the substrate on which the antenna. This makes it possible to increase effectively the bandwidth. In addition, instead of a direct coupling, there is an electromagnetic coupling via a slot machined in the ground plane [7-8].

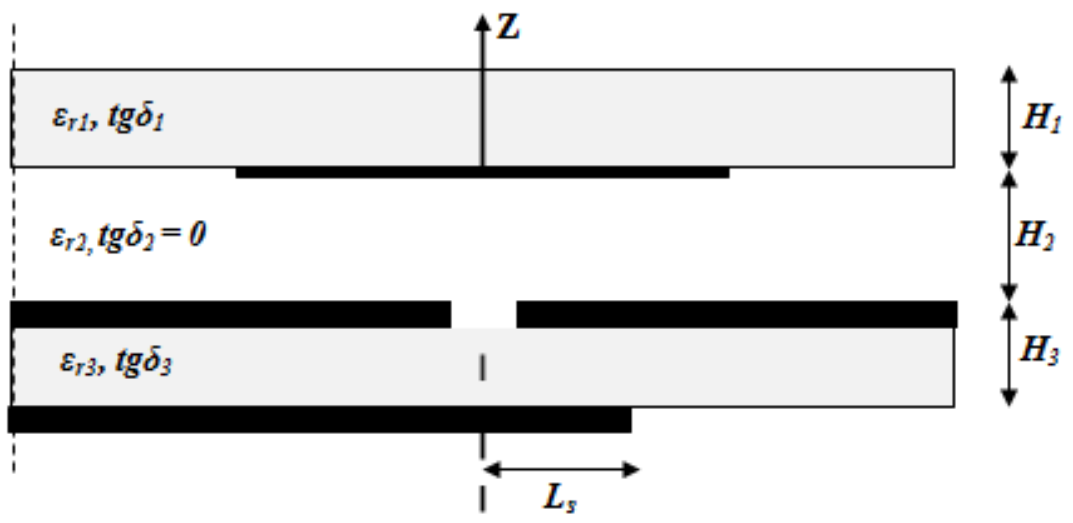

(a)

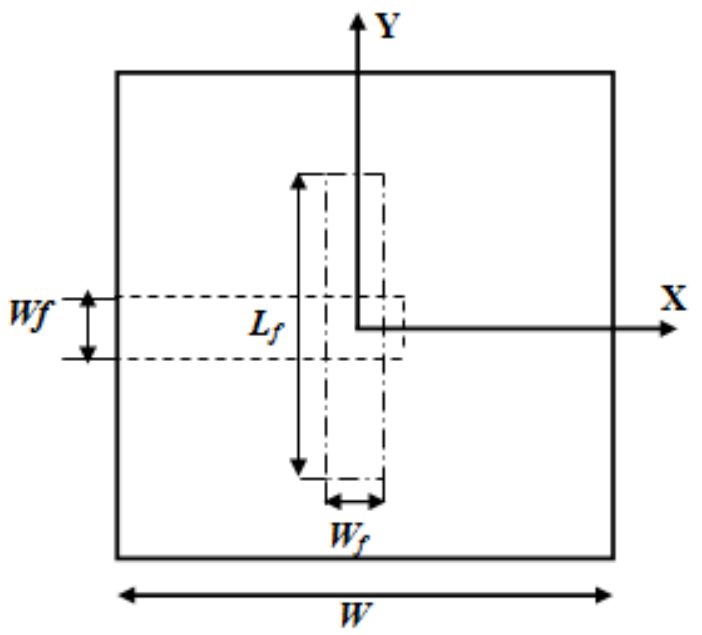

(b)

Figure 2. The used Antenna configuration

(a) Cross-sectional view, (b) top view 
The three-dimensional radiation pattern of the antenna in polar-coordinates at the frequency 2.4 $\mathrm{GHz}$ for example is presented respectively in figure 3 (a) and (b).

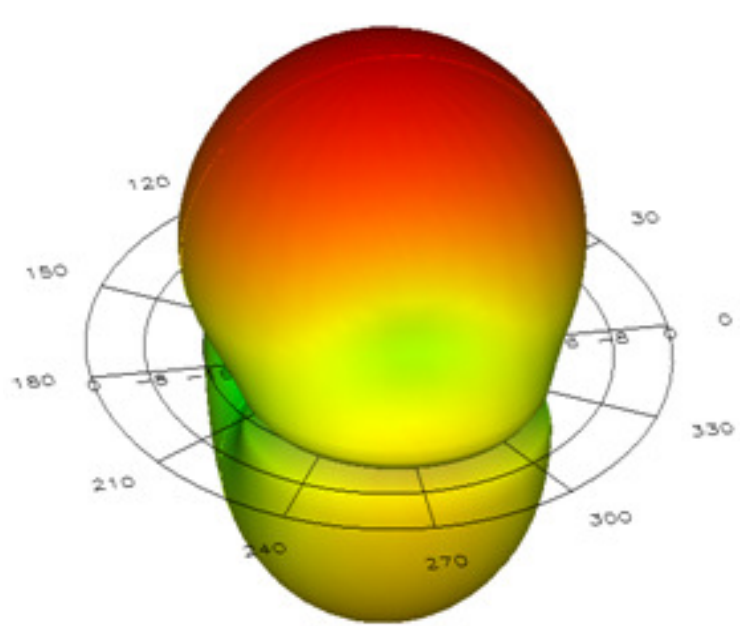

(a)

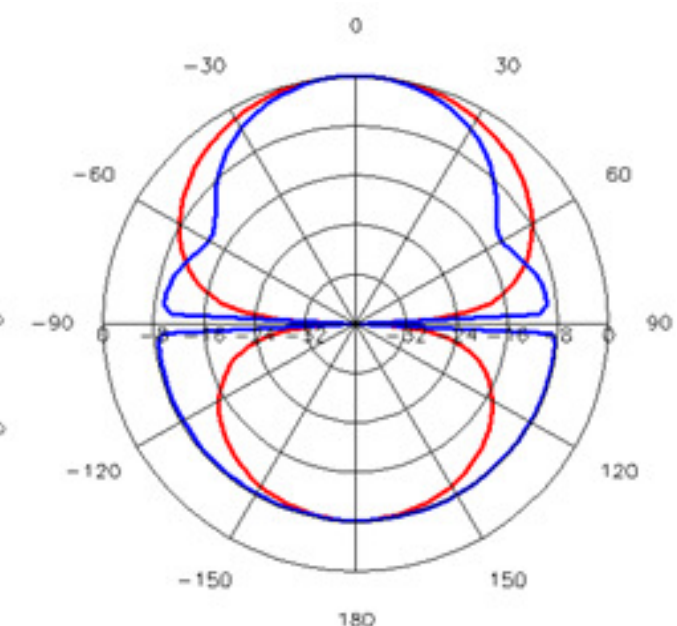

(b)

Figure 3. Radiation pattern.

(a) in $3 \mathrm{D}(f=2.4 \mathrm{GHz})$

(b) in polar-coordinates $(f=2.4 \mathrm{GHz})$

EPlane $\left(\varphi=0^{\circ}\right)$

H Plane $\left(\varphi=90^{\circ}\right)$

These graphs show the radiated power from the alone antenna at the center frequency $2.4 \mathrm{GHz}$, there is a single lobe whose maximum power is pointed in the direction $\theta=0^{\circ}$ for the $\mathrm{E}$ plane and $\mathrm{H}$ plane with an absence of side lobes. The same remark for the back plane but with a slightly smaller power, this means that our type of antenna produces back radiation due to the presence of the ground plane in the middle where the coupling slot has been machined.

\section{LOG PERIODIC ANTENNAS ARRAY}

These arrays are designed to operate at the desired frequency band [4.5 to $6.5 \mathrm{GHz}$ ]. A series feed has been applied which consist of using a straight printed line with branches to each radiating element.

The antennas array characteristics are:

- The radiating elements (patches) are of square shape of side $\mathrm{W}$, printed under substrate with a relative permittivity $\varepsilon_{1}=2.2$, of height $\mathrm{H}_{1}=1.52 \mathrm{~mm}$ and loss tangent $\operatorname{tg} \delta 1=0.001$.

- An air layer of height $\mathrm{H}_{2}=15 \mathrm{~mm}$ located below the radiating elements to increase the bandwidth.

- A power supply via a coupling slot of length $\mathrm{L}_{\mathrm{f}}$ and width $\mathrm{W}_{\mathrm{f}}$ located in the ground plane.

- A microstrip line of width $\mathrm{W}_{\mathrm{f}}$, characteristic impedance $\mathrm{Zc}=50 \Omega$ terminated by a stub Ls. This line, located below the ground plane, based on a substrate layer of relative permittivity $\varepsilon \mathrm{r}_{3}=2.2$, height $\mathrm{H}_{3}=$ of $0.762 \mathrm{~mm}$, and loss tangent $\operatorname{tg} \delta_{3}=0.001$. 


\subsection{Five elements antennas array}

Table 1 gives the resonance frequencies and the dimensions of each radiating element. The periodicity factor is chosen such that: $\tau=1.09$.

\begin{tabular}{|c|c|c|}
\hline Element & Frequency [GHz] & W = L [mm] \\
\hline 1 & 4.54 & 26.12 \\
2 & 4.95 & 23.96 \\
3 & 5.40 & 21.98 \\
4 & 5.87 & 20.17 \\
5 & 6.41 & 18.50 \\
\hline
\end{tabular}

Table 1. Frequency and dimensions of the radiating elements.

Figure 4 shows the input reflection coefficient for each antenna. Analysis of these curves thus obtained, shows a good matching at the resonance frequency, because the amplitude level of the coefficients is less than $-30 \mathrm{~dB}$.

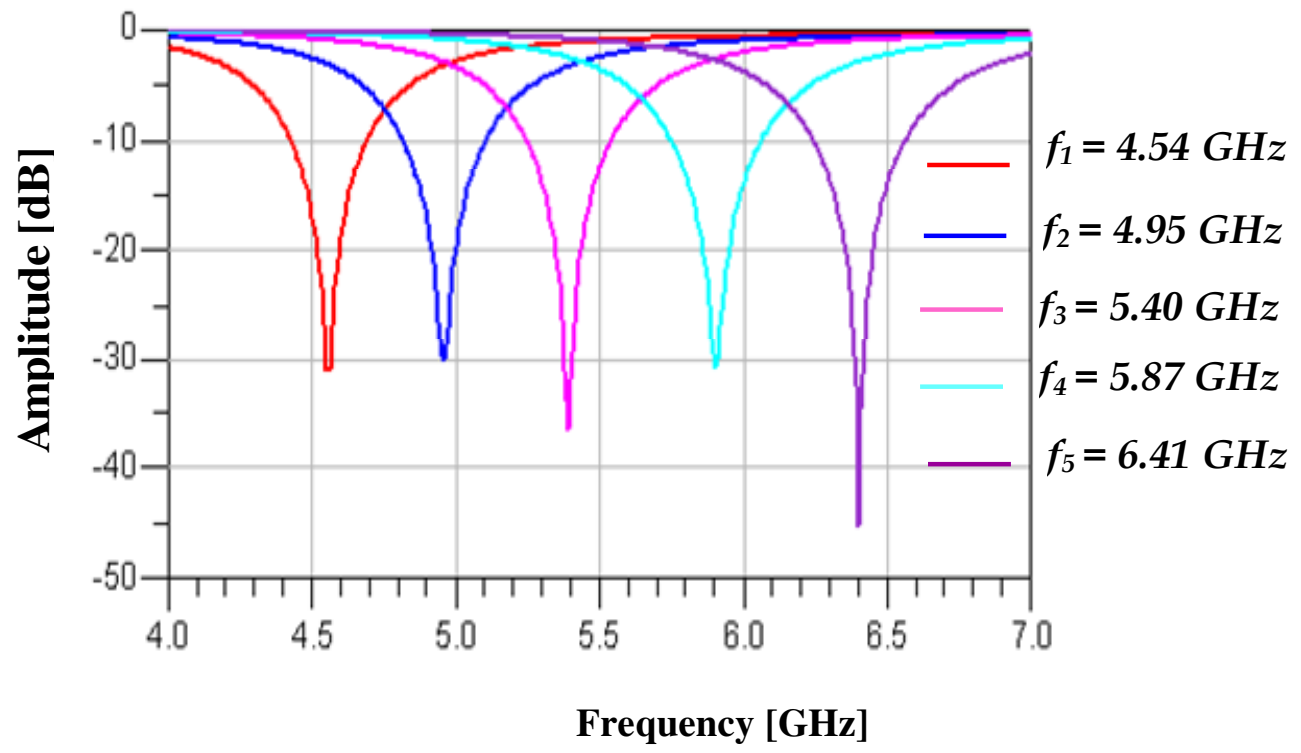

Figure 4. The Computed input reflection coefficient for each antenna.

The mask layout of the matched antennas array is represented by figure 5. The antennas array is fed via an SMA connector. 


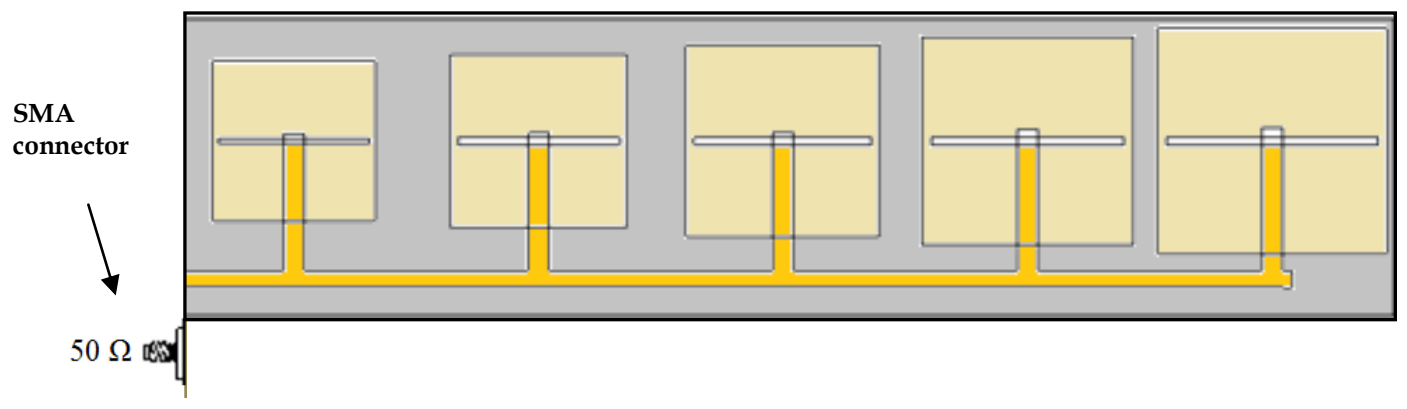

Figure 5. The mask layout of the antennas array composed of 5 radiating elements.

Figure 6 shows the input return loss of the antennas array for $4 \mathrm{GHz}$ to $7 \mathrm{GHz}$.

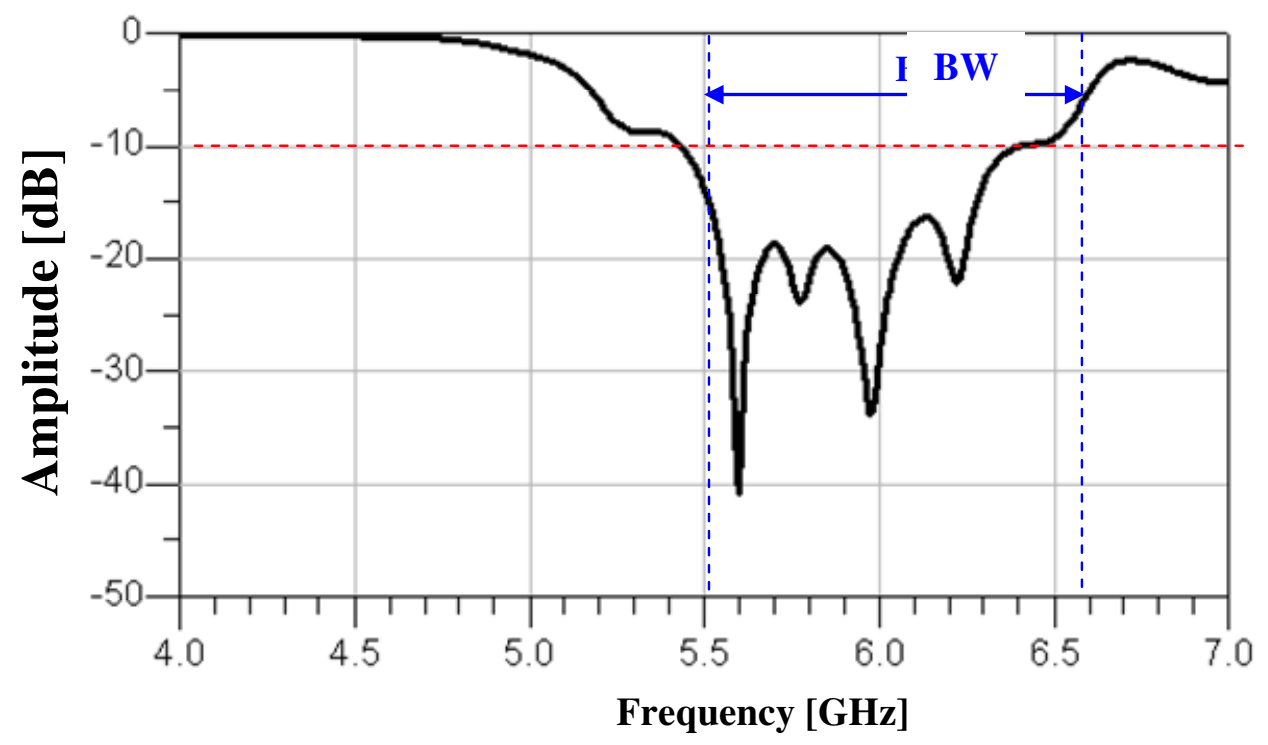

Figure 5. The Computed input return loss of the antennas array composed of 5 radiating elements.

The examination of this result highlights the appearance of a large bandwidth between $5.45 \mathrm{GHz}$ and $6.5 \mathrm{GHz}$. This bandwidth is of the order of $17.96 \%$. The antennas array is well matched. 


\subsection{Seven elements antennas array}

On Table 2, we give the resonance frequencies and the dimensions of each radiating element. The total number of elements is 7 . The periodicity factor is chosen such that: $\tau=1.06$.

\begin{tabular}{|c|c|c|}
\hline Element & Frequency [GHz] & $\mathbf{W}=\mathbf{L}[\mathbf{m m}]$ \\
\hline 1 & 4.54 & 26.12 \\
2 & 4.80 & 24.66 \\
3 & 5.09 & 23.28 \\
4 & 5.39 & 21.98 \\
5 & 5.71 & 20.07 \\
6 & 6.05 & 19.59 \\
7 & 6.41 & 18.49 \\
\hline
\end{tabular}

Table 2. Frequency and dimensions of the radiating elements

On figure 4, we represent the input return losses for each antenna from the $4 \mathrm{GHz}$ to $7 \mathrm{GHz}$.

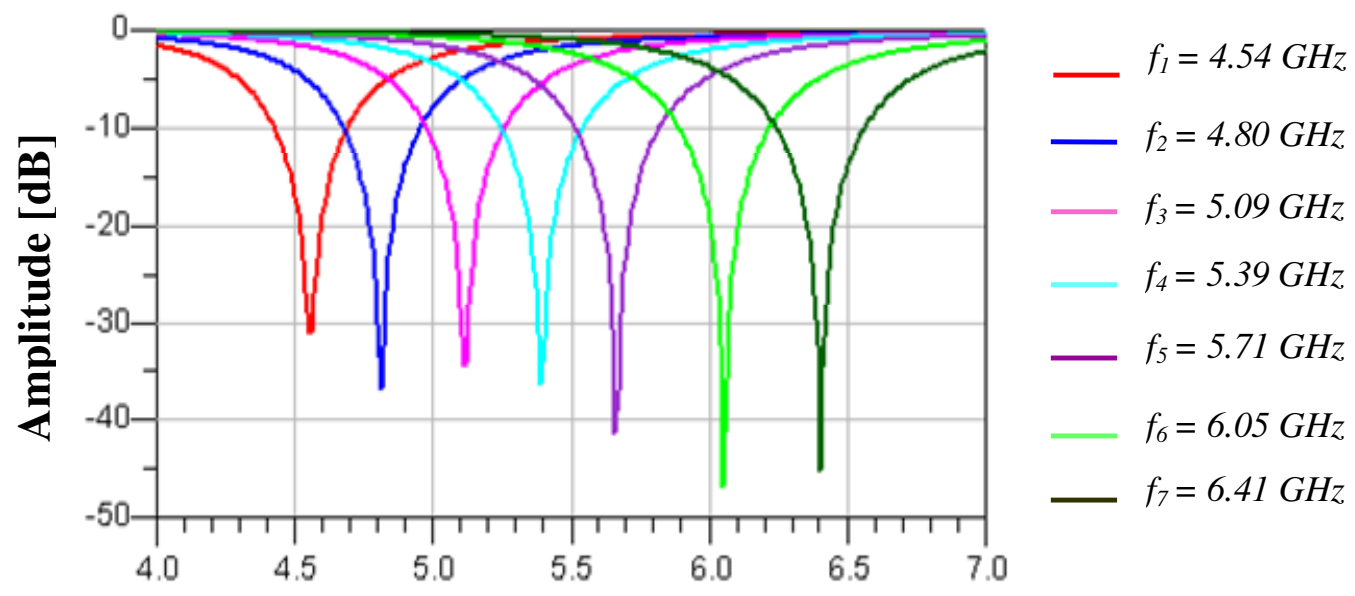

Frequency $[\mathbf{G H z}]$ 
International Journal of Distributed and Parallel Systems (IJDPS) Vol.3, No.3, May 2012

Figure 6. Computed input return losses for each antenna

As shown in Figure 7, the amplitude of the reflection coefficient at the resonance frequency of each antenna is less than $-30 \mathrm{~dB}$. This implies that the antennas have a very good matching.

The mask of the antennas array composed of 7 elements is shown in Figure 8.

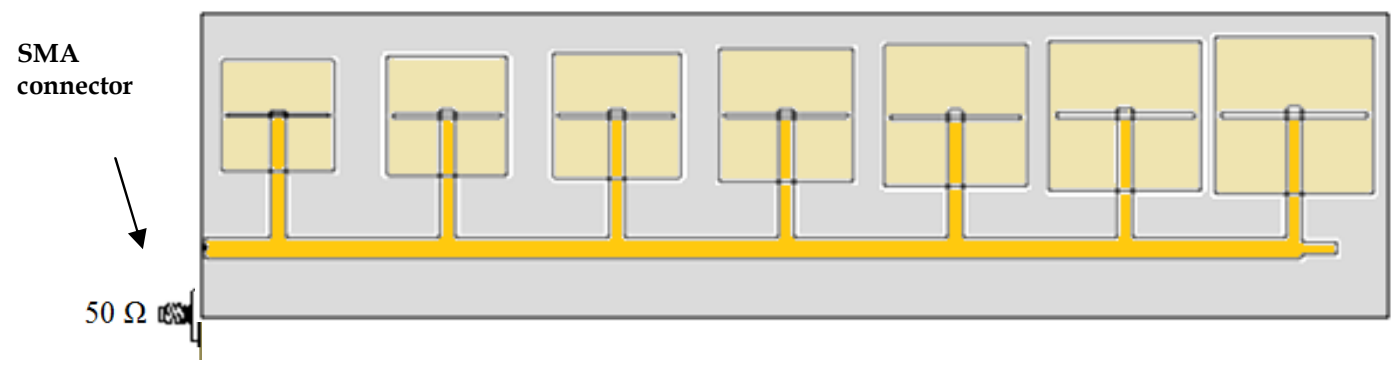

Figure 7. The mask layout of the antennas array composed of 7 radiating elements

The input return loss of the antennas array composed of 7 elements for $4 \mathrm{GHz}$ to $7 \mathrm{GHz}$ is depicted in Figure 9.

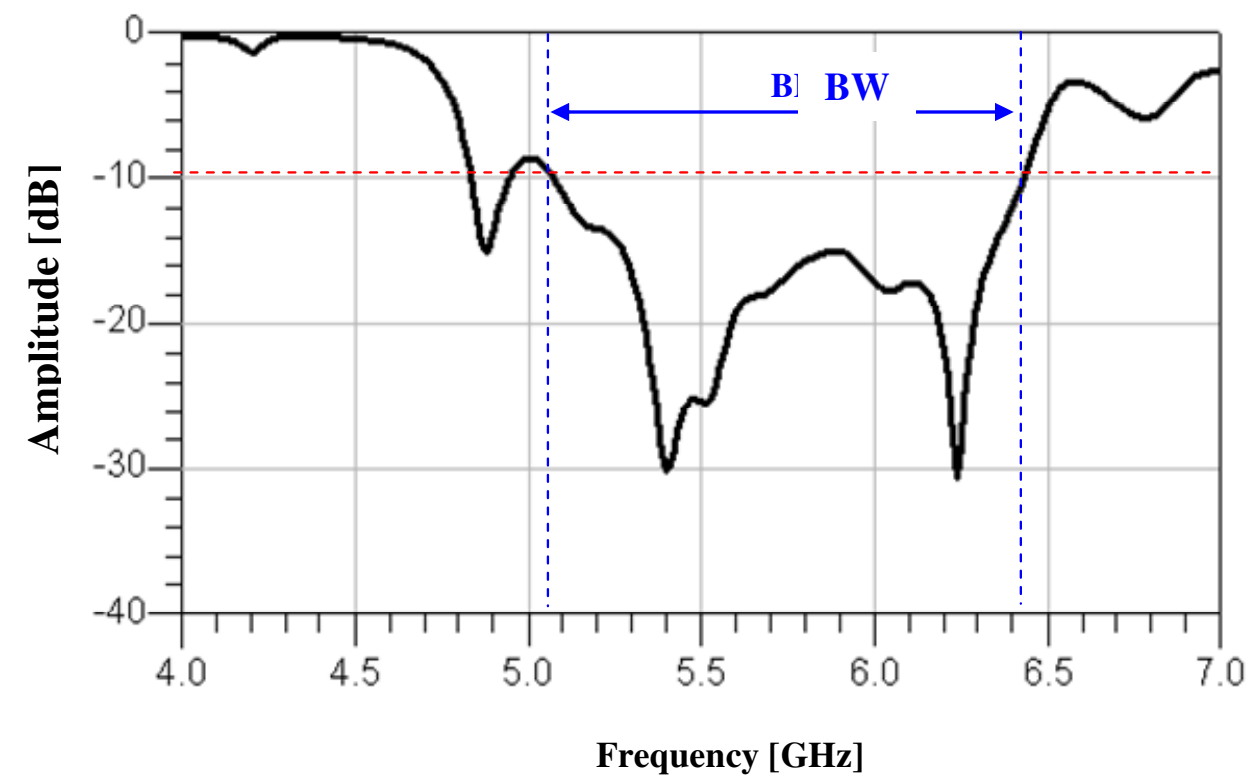

Figure 8. The Computed input return loss of the antennas array composed of 7 radiating elements 
International Journal of Distributed and Parallel Systems (IJDPS) Vol.3, No.3, May 2012

Notice from figure 9, a bandwidth between $5.07 \mathrm{GHz}$ and $6.43 \mathrm{GHz}$. This band is larger $(23.65 \%)$ if we compared to that found for the antennas array composed of five elements $(17.96 \%)$.

\subsection{Nine Elements Antennas Array}

On Table 3, we give the resonance frequencies and the dimensions of each radiating element. The total number of elements in this case is chosen equal to 9 . The periodicity factor is chosen such that: $\tau=1,044$.

\begin{tabular}{|c|c|c|}
\hline Element & Frequency [GHz] & W = L [mm] \\
\hline 1 & 4.54 & 26.12 \\
2 & 4.74 & 25.01 \\
3 & 4.95 & 23.96 \\
4 & 5.18 & 22.95 \\
5 & 5.40 & 21.98 \\
6 & 5.65 & 21.06 \\
7 & 5.87 & 20.17 \\
8 & 6.17 & 13.32 \\
9 & 6.41 & 18.50 \\
\hline
\end{tabular}

Table 3. Frequency and dimensions of the radiating elements

In figure 10, we depict the input return loss amplitudes result of each radiating element.

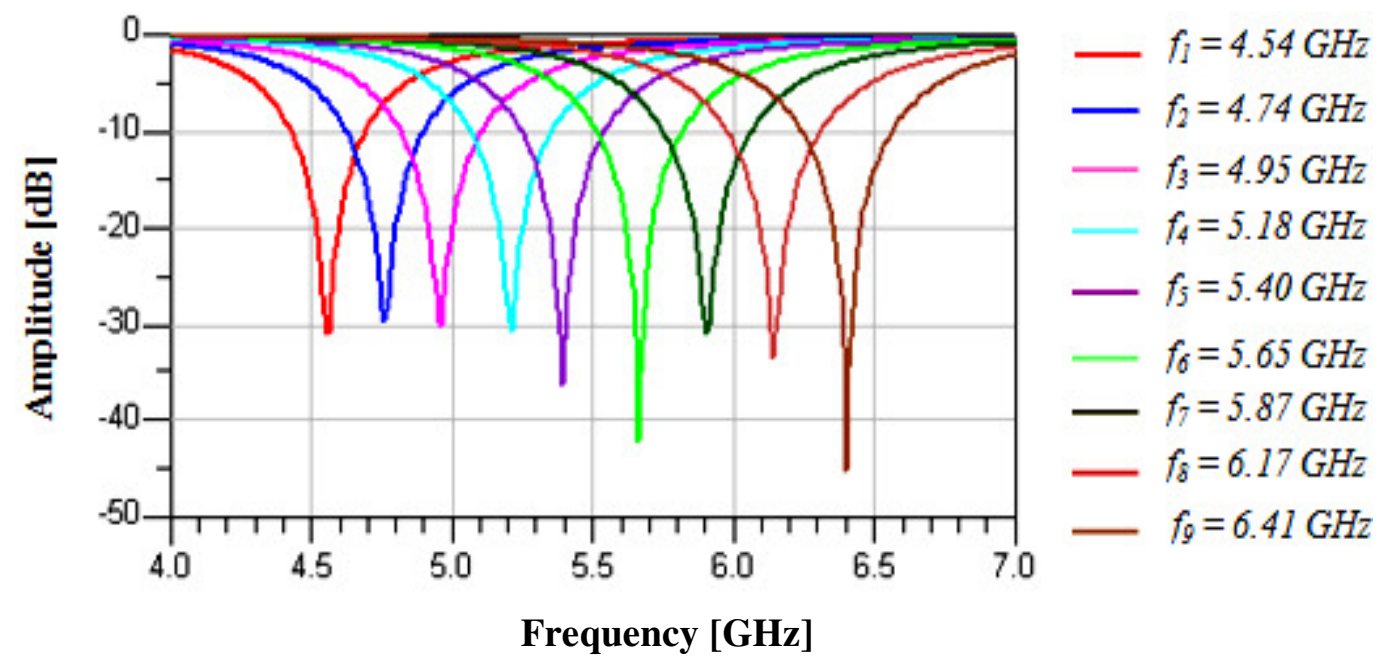


International Journal of Distributed and Parallel Systems (IJDPS) Vol.3, No.3, May 2012

Figure 9. Computed input return losses for each antenna

On this plot, it appears an amplitude level of the reflection coefficient below $-30 \mathrm{~dB}$ at the resonant frequency of each antenna; this shows the very good adaptation of antennas. The mask layout of the antennas array composed of 9 elements to is represented by Figure 11.

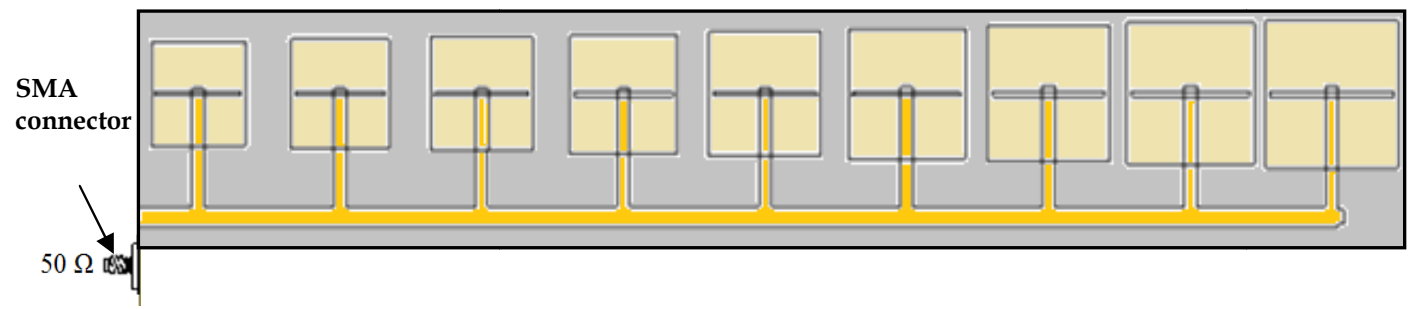

Figure 10. The mask layout of the antennas array composed of 9 elements.

Bandwidth and the input reflection coefficient of antennas array are shown in figure 12.

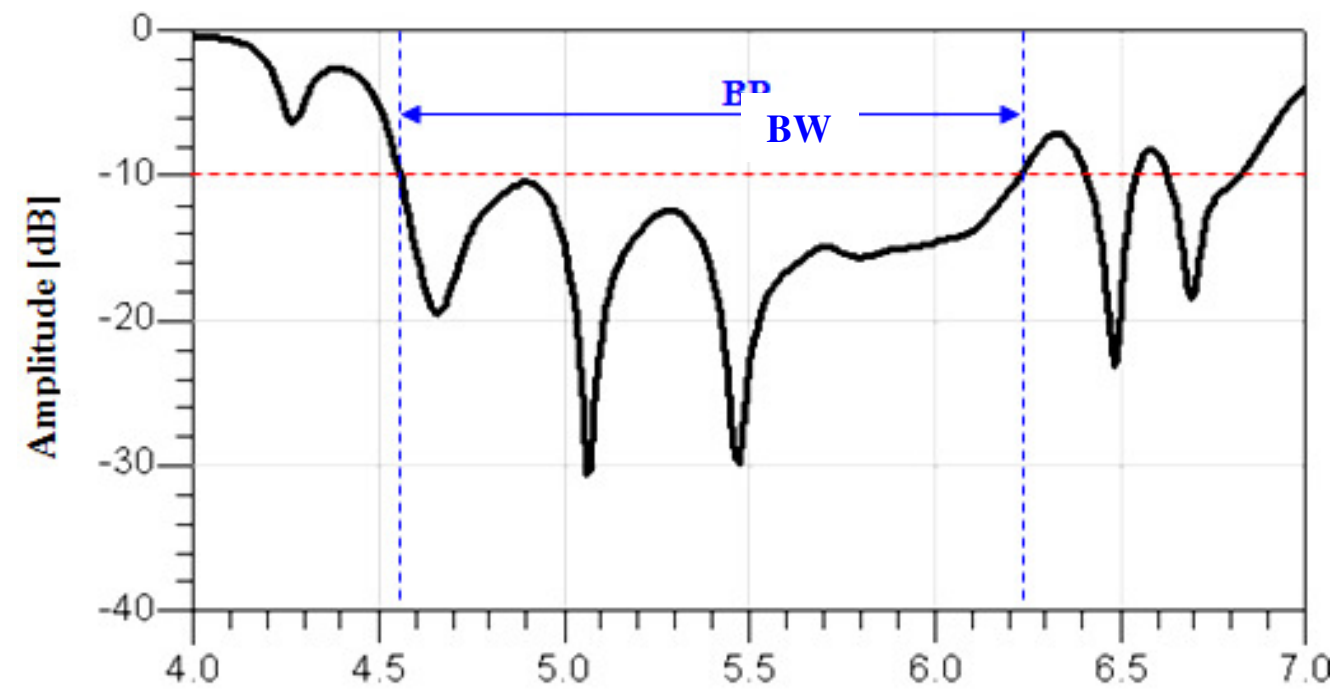

Frequency [GHz] 
International Journal of Distributed and Parallel Systems (IJDPS) Vol.3, No.3, May 2012

Figure 11. The Computed input return loss of the antennas array composed of 9 radiating elements

The results show in explicit manner that the gain increases proportionally with the number of elements. The observation of the simulation results in terms of return loss shown in figure 12 , brings up a working bandwidth between $4.54 \mathrm{GHz}$ and $6.24 \mathrm{GHz}$. This result is better (31.53\%) if it is compared to that of an array of 5 and 7 elements.

In order to observe the gain evolution over the C-band versus the number of elements, we chose the $5.5 \mathrm{GHz}$ frequency; the results are depicted in Figure 13.

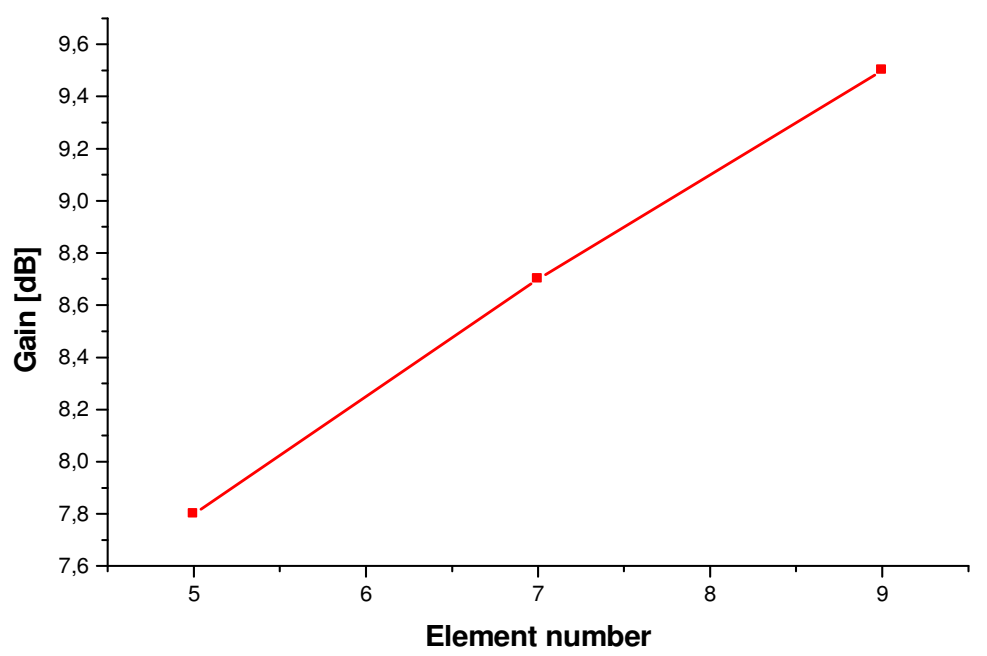

Figure 12. Variation of the gain versus the number of radiating elements at the frequency

\section{Conclusion}

$5.5 \mathrm{GHz}$

In this paper we have presented the simulation results of log periodic antenna arrays fed by microstrip line through a slot. Simulation results are obtained by a rigorous method (the moment's method). The examination of the results shows a good improvement in the gain and bandwidth. We have found that the bandwidth increases when the number of elements constituting the array increases. Finally we have seen the advantage of having a log-periodic structure with a high number of elements, a bandwidth of about $1700 \mathrm{MHz}(31.53 \%)$ was obtained by an array of nine elements by comparing that of the antennas array composed of 7 and 5 respectively which are respectively of about $1300 \mathrm{MHz}(23.65 \%)$ and $1050 \mathrm{MHz}$ $(17.96 \%)$.

\section{REFERENCES}

[1] Aanandan. CK, Nair KG (1986) 'Compact broadband microstrip antenna'. Electron Lett 22:10641065.

[2] Hongming A, Nauwelaers. KJC, Van de Capelle AR (1994) 'Broadband active microstrip antenna design with the simplified real frequency technique'. IEEE Trans Antennas Propag 42:1612-1619. 
International Journal of Distributed and Parallel Systems (IJDPS) Vol.3, No.3, May 2012

[3] Romodin. V. b, Oznobikhin. V. I and Kopylov. V. V, 'Log Periodic Microstrip Array', IEEE-Russia conference, MIA-ME '99m 1999.

[4] Kakar. R and Kumar. G, ' Stagger Tuned Microstrip Log Periodic Antenna', IEEE conference, 1996.

[5] Rahim. M. K. A and Gardner. P. The design of Nine Element Quasi Microstrip Log-periodic Antenna. IEEE RF and Microwave Conference 2004, 5 -6 October 2004, pp. 132- 135.

[6] Rahim. M. K. A, Ahmad. M. R, Asrokin. A and Aziz. M. Z. A. A. 'The Design of UWB antenna using log Periodic Technique. Loughbrough Antennas and Propagation Conference (LAPC 2006), 2nd - 3rd April 2006, Loughbrough, U.K.

[7] Pozar. D. M., "Mcrostrip Antenna Aperture Coupled to a Microstrip Line "., Elect Letters, vol. 21, pp. 49--50, Jan. 1985.

[8] D. H. Shaubert., Pozar. D. M and Adrian. A., "Effects of Microstrip Antenna Substrate Thickness and Permittivity : Comparison of Theories with Experiment", IEEE- trans. AP, vol 37, $\mathrm{n}^{\circ}: 6$, june 1989, p.677-782. 